\title{
Hollow sphere, a flexible multimode Gravitational Wave antenna
}

\author{
J Alberto Lobo \\ Departament de Física Fonamental, Universitat de Barcelona, Diagonal 647, \\ 08028 Barcelona, Spain.
}

\begin{abstract}
Hollow spheres have the same theoretical capabilities as the usual solid ones, since they share identical symmetries. The hollow sphere is however more flexible, as thickness is an additional parameter one can vary to approach given specifications. I will briefly discuss the more relevant properties of the hollow sphere as a GW detector (frequencies, cross sections), and suggest some scenarios where it can generate significant astrophysical information
\end{abstract}

PACS numbers: PACS numbers: 04.80.Nn, 95.55.Ym, 04.30.Nk

\section{Introduction}

A Gravitational Wave (GW) is a time-dependent perturbation of the background space-time geometry which propagates away from the source, and which is generated by changes in the mass-energy distribution of the latter. Due to the weakness of gravitation, the mathematical description of GWs, so far as detection by a remote observatory is concerned, can be very accurately accomplished by means of a linear approximation in the field equations. More precisely, if $\eta_{\mu \nu}$ is the (Lorentzian) background metric, the actual metric $g_{\mu \nu}(\mathbf{x}, t)$ can be expediently split up as

$$
g_{\mu \nu}(\mathbf{x}, t)=\eta_{\mu \nu}+h_{\mu \nu}(\mathbf{x}, t), \quad\left|h_{\mu \nu}\right| \ll 1
$$

where $\mathbf{x}$ and $t$ are quasi-Lorentzian coordinates, and the $h_{\mu \nu}(\mathbf{x}, t)$ are the GW perturbations.

Since GWs will of course be observed far from their source they can be safely assumed to be plane across the detector's span. In this case only the six so called electric components, $R_{0 i 0 j}(\mathbf{x}, t)$, of the Riemann tensor associated with (传) are not identically zero. Although General Relativity theory predicts that at least four of these are zero, as explained in textbooks [1], alternative theories of gravity (e.g. Brans-Dicke) are generally less restrictive - see [2, 3] for a complete discussion of all the possibilities. It thus appears that detection of GWs is a strong experimental test on which is the theory which best describes gravity.

Currently operating Weber bars, as well as interferometric detectors, such as LIGO and VIRGO, are however single channel devices, i.e., they can only sense one linear combination of the 5 quadrupole GW amplitudes - the so called antenna pattern [4, 5]. A network of such detectors in joint operation is thus necessary for a complete deconvolution of the GW signal, but this poses non-trivial practical problems 
of analysis, because there are no two identical devices, noise characteristics change from one to the other, etc.

A simple way out of such limitation is to use a spherical detector, because this is a natural multimode, or tensor, GW antenna 6, 7. 8. . The reason for this is that, having spherical symmetry, an elastic solid has oscillation eigenmodes which come in degenerate $l$-pole harmonics, the monopole $(l=0)$ and quadrupole ones efficiently coupling to the incoming GW [9]. By use of suitable sensor systems [10, 11] it is theoretically possible to completely deconvolve the GW signal, i.e., to measure all six amplitudes which characterise a general metric GW [3].

A hollow sphere is an interesting variation of the solid one 12 which has a number of interesting capabilities as a GW detector, and flexibility to implement them by adjusting the thickness parameter. In this paper I intend to summarise the most relevant features of an ideal hollow spherical GW antenna (i.e., I will assume that it is technically possible to build a working system), and see what interesting physics could be done with it.

\section{Response and sensitivity}

As usual (see [8] for a thorough discussion), the solid's response to a GW excitation is given by the solution, with appropriate boundary conditions, to the partial differential equation

$$
\varrho \frac{\partial^{2} \mathbf{u}}{\partial t^{2}}-\mu \nabla^{2} \mathbf{u}-(\lambda+\mu) \nabla(\nabla \cdot \mathbf{u})=\mathbf{f}(\mathbf{x}, t)
$$

where $\varrho, \lambda$ and $\mu$ are the density and elastic Lamé coefficients, respectively, and

$$
\mathbf{f}(\mathbf{x}, t)=\mathbf{f}^{(00)}(\mathbf{x}) g^{(00)}(t)+\sum_{m=-2}^{2} \mathbf{f}^{(2 m)}(\mathbf{x}) g^{(2 m)}(t)
$$

is the tidal GW driving density of force. The hollow sphere's response is then given by

$$
\mathbf{u}(\mathbf{x}, t)=\sum_{n=1}^{\infty} \frac{a_{n 0}}{\omega_{n 0}} \mathbf{u}_{n 00}(\mathbf{x}) g_{n 0}^{(00)}(t)+\sum_{n=1}^{\infty} \frac{a_{n 2}}{\omega_{n 2}}\left[\sum_{m=-2}^{2} \mathbf{u}_{n 2 m}(\mathbf{x}) g_{n 2}^{(2 m)}(t)\right]
$$

where $\omega_{n l}$ are the oscillation eigenfrequencies of the $\mathbf{u}_{n l m}(\mathbf{x})$ eigenmode, $a_{n l}$ are overlap coefficients and $g_{n l}^{(l m)}(t)$ is the convolution between the signal $g^{(l m)}(t)$ and the detector mode. Eigenmode wave functions $\mathbf{u}_{n l m}$ are somewhat complicated functions which are calculated in detail in reference [12], while the frequencies of the first two quadrupole and first monopole harmonics are plotted in figure 11 for a conceivable materials and dimensions of the system.

The most relevant parameter characterising the solid's sensitivity to GWs is the cross section for the absorption of incoming GW energy. As shown in reference [12], the following formula holds for quadrupole modes:

$$
\sigma_{n} \equiv \sigma_{\mathrm{abs}}\left(\omega_{n 2}\right)=F_{n}\left(\sigma_{P} ; a / R\right) \frac{G M v_{s}^{2}}{c^{3}}
$$

where $v_{s}$ is the speed of sound in the material, $\sigma_{P}$ its Poisson ratio, and $F_{n}\left(\sigma_{P} ; a / R\right)$ is a dimensionless parameter associated with the $n$-th quadrupole mode of the hollow sphere. A plot of the first two $F_{n}$ 's is given in figure 2. The crossing 


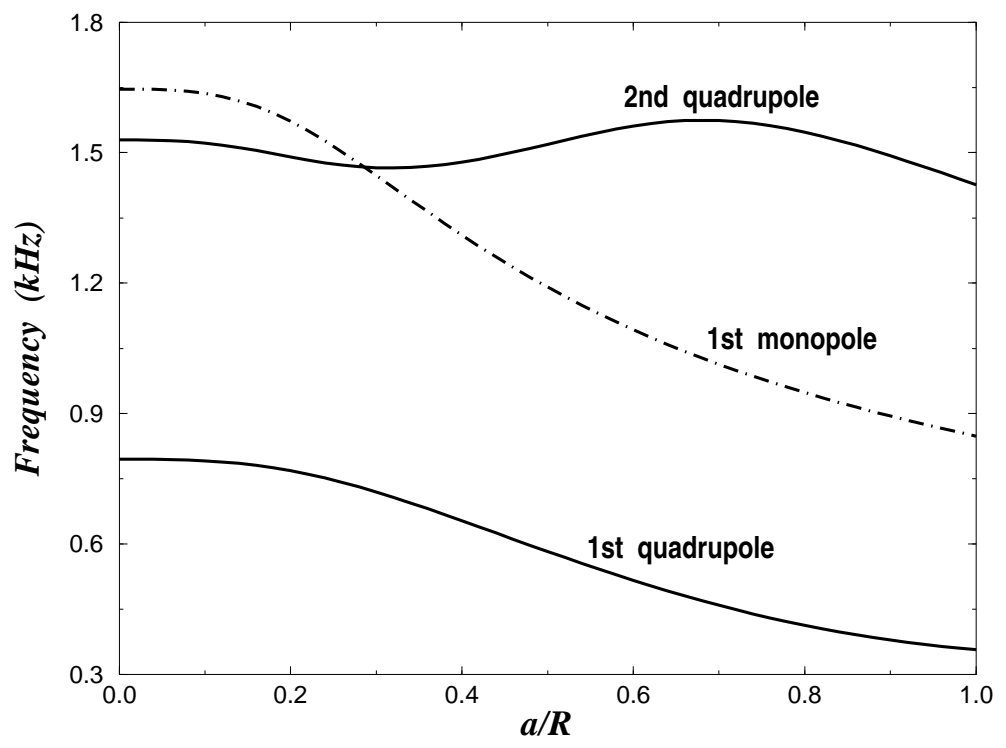

Figure 1. The first two quadrupole (solid lines) and first monopole (dash-dotted) frequencies of a hollow sphere of a $\mathrm{CuAl}$ alloy, 3 metres of outer diameter, as a function of the ratio between the inner radius, $a$, and the outer radius, $R$.

between the curves happens at $a / R=0.375$, which means a hollow sphere is a more efficient GW energy absorber in its second mode than in the first, which favours in turn higher frequency signal reception.

\section{Physics with a hollow sphere}

Let us now briefly assess which sources of GWs can be visible with a hollow spherical detector, under the ideal assumption that technological problems are solved to the effect that the detector's quantum limit has been reached. If such is the case then peak sensitivity is given by 12 .

$$
S_{h}\left(\omega_{n}\right)=\frac{G \hbar}{c^{3}} \frac{4 \pi \beta_{n}}{\sigma_{n}}
$$

where $\omega_{n}$ is a quadrupole resonance frequency, and $\beta_{n}$ is the efficiency of the coupling between mechanics and electronics in that mode; the detector's bandwidth is $\Delta \omega_{n}=\beta_{n} \omega_{n}$.

Take for example a hollow sphere of $\mathrm{CuAl}, 3$ metres of outer diameter and 22 centimetres thick $(a / R=0.15)$, weighing 40 tons; the first two quadrupole frequencies are then 400 and $1500 \mathrm{~Hz}$, respectively, and the peak sensitivity is essentially identical in both modes, reaching $10^{-46} \mathrm{~Hz}^{-1}$ for a $\beta$ factor of 0.1 . A system like this would be able to see a millisecond burst with an amplitude of $10^{-22}$ metres/metre, and a monochromatic signal as weak as $10^{-27}$ metres/metre at the first resonant frequency if integrated for one year. Such sensitivities would most likely enable the observations of supernova explosions in galaxies out to the Virgo cluster (at a rate of a few per year), and a significant sample of the galactic pulsar population. 


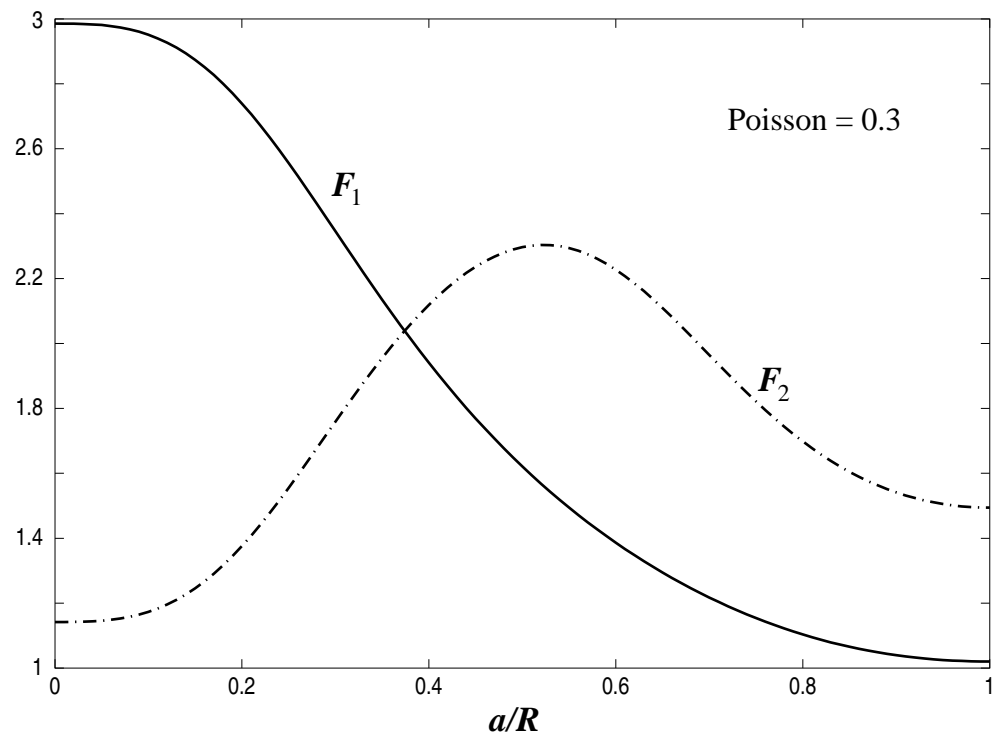

Figure 2. The first two quadrupole cross section coefficients of equation (5) for hollow spheres of a $\mathrm{CuAl}$ alloy. In abscissae the ratio between the inner radius, $a$, and the outer radius, $R$. This plot is of course independent of the antenna's absolute size.

A larger detector, 6 metres in outer diameter, 25 centimetres thick, 200 tons of $\mathrm{CuAl}$ alloy, has a minimum spectral density of noise of $3 \times 10^{-47} \mathrm{~Hz}^{-1}$ at its first resonance $(190 \mathrm{~Hz})$, and $2 \times 10^{-47} \mathrm{~Hz}^{-1}$ at the second $(750 \mathrm{~Hz})$. Such a system can be used for chirp signal detection by a robust method first described for spherical detectors by Coccia and Fafone 13. The idea is conceptually simple: the GW signal generated e.g. by a compact binary system nearing coalescence sweeps an ascending frequency range which eventually overlaps with the sensitivity bands of the detector. If the binary remains undisrupted [14] while in the frequency intervals between the first two just quoted resonances then the antenna's response will peak at those frequencies, with a signal to noise ratio given by

$$
S N R=\frac{5 \pi 2^{1 / 3}}{24} \frac{\left(G M_{c}\right)^{5 / 3}}{c^{3}} \frac{\Delta \omega_{n}}{S_{h}\left(\omega_{n}\right)} \frac{1}{r^{2} \omega_{n}^{7 / 3}}
$$

where $r$ is the distance to the source, $\omega_{n}$ is a detector quadrupole resonance frequency, and $M_{c}$ is the mass parameter

$$
M_{c} \equiv\left(m_{1} m_{2}\right)^{3 / 5}\left(m_{1}+m_{2}\right)^{-1 / 5}
$$

of the chirp signal. Let now $\tau_{1}$ and $\tau_{2}$ be the times at which the the signal reaches the detector with frequencies $\omega_{1}$ and $\omega_{2}$, respectively. The mass parameter can then be estimated by the formula

$$
M_{c}=\frac{5^{3 / 5}}{2^{16 / 5}} \frac{c^{3}}{G}\left(\frac{\omega_{2}^{-8 / 3}-\omega_{1}^{-8 / 3}}{\tau_{2}-\tau_{1}}\right)^{3 / 5}
$$

in which the lowest post-Newtonian approximation for the waveform has been assumed [13]. The strong point of this double passage method is its robustness 
relative to the details of the signal's phase [15, 16], which considerably complicates the performance of the matched filter in extracting the signal from the noise in an interferometric detector [17]. It thus appears that both detectors, a sphere and an interferometer, can constructively cooperate in the identification of compact binary coalescences, as the sphere can provide a good estimate of the mass parameter which will simplify and enhance the efficiency of the bank of filters needed in an interferometer [17].

The hollow sphere is particularly suited for this, as it can be made to have low resonance frequencies, and this favours SNR because the signal stays longer in the lower frequencies - gravitational bremsstrahlung tends to accelerate the inspiral as the binary's period of revolution diminishes. This fact is clearly reflected by equation (7), where SNR is seen to strongly depend on the $7 / 3$ inverse power of frequency.

Cosmic backgrounds of GWs can also be observed with hollow spheres. Such signals, due to their stochastic nature, are impossible to tell from the detector noise. A minimum of two detectors, whose outputs are cross-correlated, must be used in order to see a common signal which can be identified by a distinctive spectral density, $S_{\mathrm{GW}}(\omega)$. With unit SNR, a pair of identical hollow spheres could set a good limit on the density parameter of GWs in the Universe [21, 12]:

$$
\Omega_{\mathrm{GW}} \simeq 10^{-9} \times\left(\frac{f_{n}}{200 \mathrm{~Hz}}\right)^{3}\left(\frac{S_{h}^{(1)}\left(f_{n}\right) S_{h}^{(2)}\left(f_{n}\right)}{10^{-96} \mathrm{~Hz}^{-2}}\right)^{1 / 2}\left(\frac{20 \mathrm{~Hz}}{\Delta f_{n}}\right)^{1 / 2}\left(\frac{1 \text { year }}{\tau}\right)^{1 / 2}
$$

where $\tau$ is the integration time and $\Delta f_{n}$ the detector's bandwidth around $f_{n}$.

It has recently been conjectured by Amelino-Camelia [18], on the basis of dimensional arguments, that a cosmic background of GWs could have, in first approximation, a flat spectrum with spectral density

$$
S_{\mathrm{GW}}(\omega) \sim \frac{\ell_{\mathrm{Planck}}}{c}=\left(\frac{G \hbar}{c^{5}}\right)^{1 / 2} \sim 5 \times 10^{-44} \mathrm{~Hz}^{-1}
$$

It is unclear whether this is a realistic assumption [19]; if it were however it would mean that Planck scale scale physics would become accessible to the next generation of GW detectors, and hollow spheres would enable the observation of such phenomena with signal to noise ratios of the order of about 100 .

\section{Closing remarks}

As already mentioned, spherical elastic bodies are natural multimode GW antennas. The reason for this is the degeneracy of their quadrupole oscillation eigenmodes, which ideally match the structure of GWs. Both a solid and a hollow sphere share this property, of course, and in this paper I have briefly considered some of the more interesting properties of the latter, related to its sensitivity and subsequent capabilities as a powerful GW telescope. I have deliberately left aside the issues of how it could be cast and suspended in practice, as well as questions of how a quantum limited transducer system can be coupled efficiently to the body of the antenna. The paper is thus intended to give a consistent flavour of the best performance one can possibly aim at with such a detector.

There being the possibility to adjust the spherical shell's thickness, the hollow sphere is a more flexible device than a solid one to meet given specifications. In fact, recent research work [20] shows that it is advantageous to use a hollow and a 
solid sphere placed in a joint concentric layout to produce a GW detector which has enhanced sensitivity over a frequency band between the resonances of the inner solid body and the outer hollow one. By an optimised choice of the latter's thickness, and by means of optical Fabry-Perot cavities acting as non-resonant transducers, such a nested sphere system appears to be a promising device for GW signal detection in the $\mathrm{kHz}$ region in bandwidths of several hundred $\mathrm{Hz}$, where it beats the prospects of the very sensitive earth based interferometric GW detectors. And, let me stress this once more, all of it with the ability to potentially deconvolve the five quadrupole amplitudes with a single system.

\section{Acknowledgements}

I acknowledge with thanks support from the Spanish Ministry of Education, contract code BFM2000-0604.

\section{References}

[1] C.W. Misner, K.S. Thorne, J.A. Wheeler, Gravitation, Freeman 1973.

[2] D.M. Eardley, D.L. Lee \& A.P. Lightman, Phys. Rev. D 8, 3308 (1973)

[3] C.M. Will, Theory and experiment in Gravitational Physics, Cambridge University Press 1993

[4] S.V. Dhurandhar \& M. Tinto, Mon. Not. Roy. Astr. Soc. 234, 663 (1988)

[5] M. Montero, PhD thesis memoir, University of Barcelona (Spain) 1998, available at ftp://fismat.ffn.ub.es/pub/PhD_theses/postscript/m_montero.zip

[6] R. Forward, Gen. Rel. and Grav. 2, 149 (1971)

[7] R.V. Wagoner \& H.J. Paik in Experimental Gravitation, Proceedings of the Pavia International Symposium, Accad. Naz. dei Lincei, pag. 257, Roma 1977

[8] J.A. Lobo, Phys. Rev. D 52, 591 (1995)

[9] E. Coccia, J.A. Lobo, J.A. Ortega, Phys. Rev. D 52, 3735 (1995)

[10] W.W. Johnson \& S.M. Merkowitz, Phys. Rev. Lett. 70, 2367 (1993)

[11] J.A. Lobo, Mon. Not. Roy. Astr. Soc. 316, 173 (2000)

[12] E. Coccia, V. Fafone, G. Frossati, J.A. Lobo \& J.A. Ortega, Phys. Rev. D 57, 2051 (1998)

[13] E. Coccia \& V. Fafone, Phys. Lett. A 213, 16 (1996)

[14] J.P.A. Clark \& D.M. Eardly, Astrophys. J. 215, 311 (1977)

[15] C. Cutler et al., Phys. Rev. Lett. 70, 2984 (1993)

[16] K.S. Thorne, also in these Proceedings

[17] A. Pai, S.V. Dhurandhar \& S. Bose, Phys. Rev. D 64, 042004 (2001)

[18] G. Amelino-Camelia, Nature 410, 1065 (2001)

[19] Pia Astone, private communication

[20] M. Cerdonio, L. Conti, J.A. Lobo, A. Ortolan, L. Taffarello \& J.P. Zendri, Phys. Rev. Lett. 87, $031101(2001)$

[21] P. Astone, J.A. Lobo \& B.F. Schutz, Class. Quant. Gravity 11, 2093 (1994) 